\title{
Retrograde cardioplegia
}

\author{
Ganghong Tian, MD, PhD, ${ }^{a}$ Bo Xiang, $\mathrm{DDS}^{\text {a }}$ Guangping Dai, PhD, ${ }^{a}$ Gang Li, MD, MSc, ${ }^{a, b}$ \\ Jiankang Sun, MSc, ${ }^{a}$ Randy Summers, BSc, ${ }^{a}$ and Roxanne Deslauriers, $\mathrm{PhD}^{\mathrm{a}}$
}

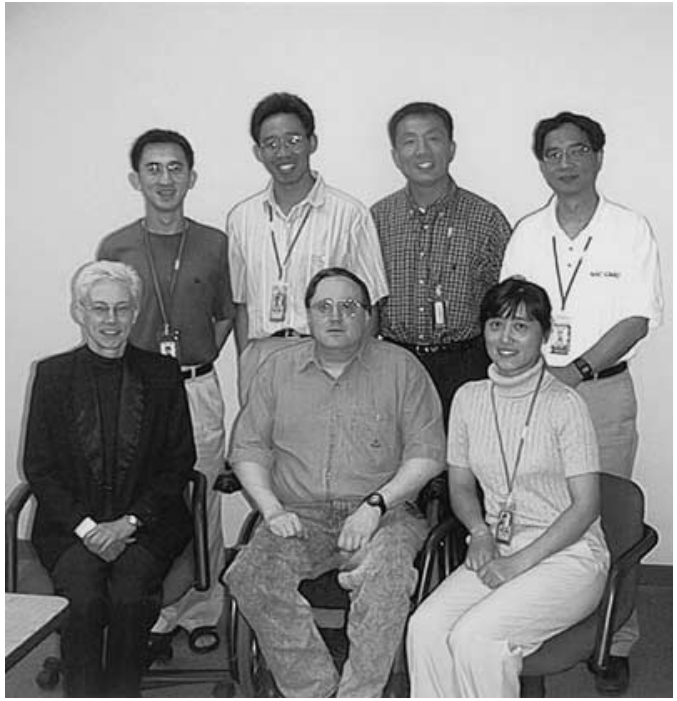

Left to right: (top) Li, Dai, Tian, Sun; (bottom) Deslauriers, Summers, Xiang
Objective: This study was undertaken to compare the efficacy of retrograde cardioplegia for myocardial perfusion with that of antegrade cardioplegia at the same flow rate.

Methods: Colored microspheres were used in rat hearts to assess the capillary flow of cardioplegia solution. Myocardial perfusion was evaluated with magnetic resonance imaging in pig hearts. Phosphorus 31 magnetic resonance spectroscopy was used to determine the efficacies of the cardioplegic techniques in sustaining myocardial energy metabolism.

Results: At the same flow rate, the number of colored microspheres delivered to the capillaries by retrograde cardioplegia $(15 \pm 1$ microspheres $/ \mathrm{mm}^{2}$ ) was significantly lower than that delivered by antegrade cardioplegia ( $29 \pm 2$ microspheres $/ \mathrm{mm}^{2}$ ). Furthermore, only $19 \% \pm 3 \%$ of the colored microspheres delivered to the capillaries by retrograde cardioplegia were found in the arteriolar portions of the capillaries, whereas most $(80 \% \pm 3 \%)$ remained in the venular portions. Moreover, magnetic resonance images showed that contrast-enhanced signal-time courses obtained from different regions of the myocardium during retrograde cardioplegia varied significantly. Localized phosphorus 31 spectra showed that retrograde cardioplegia required a higher flow rate than did antegrade cardioplegia to sustain normal myocardial energy metabolism.
From the Institute for Biodiagnostics, National Research Council of Canada, ${ }^{\mathrm{a}}$ and the Department of Physiology, University of Manitoba, ${ }^{\text {b }}$ Winnipeg, Manitoba, Canada.

Supported by the National Research Council of Canada, the Canadian Institute for Health Research, and the Heart and Stroke Foundation of Manitoba.

Received for publication Feb 5, 2002; revisions requested April 23, 2002; revisions received June 25, 2002; accepted for publication July 2, 2002.

Address for reprints: Ganghong Tian, MD, $\mathrm{PhD}$, Institute for Biodiagnostics, National Research Council, 435 Ellice Ave, Winnipeg, Manitoba, Canada R3B 1 Y6 (Email: Hong.Tian@nrc.ca).

J Thorac Cardiovasc Surg 2003;125:872-80

Copyright (C) 2003 by The American Association for Thoracic Surgery

$0022-5223 / 2003 \$ 30.00+0$

doi: $10.1067 / \mathrm{mtc} .2003 .109$
Conclusions: We conclude that retrograde cardioplegia provides significantly less capillary flow than does antegrade cardioplegia. Its microvascular perfusion varies significantly among the various small areas of the myocardium. As a result, its efficacy in sustaining normal myocardial energy metabolism is lower than that of antegrade cardioplegia.

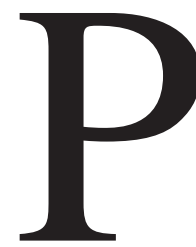
erfusion of the heart through the coronary sinus, so-called retrograde perfusion, was originally proposed by $\operatorname{Pratt}^{1}$ in 1898 . He used this approach to perfuse isolated feline hearts and was able to maintain heart contraction. His work suggested that venous perfusion had a certain degree of nutritional function. In 1956, this technique was first used by Lillehei et $\mathrm{al}^{2}$ during cardiac surgery for the protection of jeopardized myocardium because coronary atherosclerosis does not occur in the coronary venous system. ${ }^{3,4}$ Unlike antegrade cardioplegia (AC), retrograde cardioplegia (RC) avoids potential damage to the coronary arterial ostia and interferes less with surgical procedure than does AC. As a result, RC has become an alternative for heart protection during cardiac surgery. 5,6

Although RC has been used clinically, its capillary flow, regional myocardial perfusion, and efficacy in sustaining myocardial energy metabolism have not been fully established, in particular relative to AC. With radioactive microspheres in dog hearts, Partington et $\mathrm{al}^{7}$ showed that at a perfusion pressure of $50 \mathrm{~mm} \mathrm{Hg}$ the capillary flow of RC was $65 \%$ of the total cardioplegic flow. With the same approach under similar conditions, other investigators have reported quite different capillary flow ratios (from $10 \%$ to $93 \%$ ). ${ }^{8-10}$ We believe that one reason for the 
disparity may be the different sizes of microspheres used in these studies. Obviously, microspheres With different sizes could be trapped at the different sites in the microvasculature, such as the venules, draining venous capillaries, capillaries, and the Thebesian vessels. Consequently, the total numbers of microspheres trapped in various sites of the microvasculature do not provide a good measurement of capillary flow.

Colored microspheres have been used for assessment of capillary flow, flow direction, and coronary anastomoses. ${ }^{11-14}$ Because colored microspheres can be visualized under a light microscope, their distribution in the microvasculature can be readily determined in a tissue section. In this study colored microspheres in the capillaries were counted under a light microscope to assess the capillary flows of RC and AC. In addition, a capillary was divided into two anatomic segments (arteriolar and venular portions). ${ }^{11,12}$ These two portions of the capillaries were distinguished by means of histochemical techniques. ${ }^{11,12}$ Only colored microspheres distributed to the arteriolar portions of the capillaries during RC are expected to be associated With capillary flow. Those found in the venular portions of the capillaries during RC might not necessarily flow through the capillaries. Colored microspheres in the arteriolar and venular portions of the capillaries were therefore determined individually.

Most studies have used a higher perfusion pressure for $\mathrm{AC}$ than for RC when comparing them for capillary flow, ability to prevent ischemic injury, and myocardial cooling efficacy. $7,15,16$ Because the impedance of the coronary venous system is higher than that of the coronary artery system, RC would provide a significantly lower blood flow than $\mathrm{AC}$ even at the same perfusion pressure. ${ }^{17,18}$ As a result, capillary flow and ability to sustain myocardial energy metabolism of $\mathrm{RC}$ have been found to be lower than those of AC. Apparently the efficacies of RC and AC in myocardial perfusion cannot be properly evaluated at different cardioplegic flows. In this study, cardioplegia flow rate was therefore kept at the same level for both $\mathrm{RC}$ and AC.

It has been demonstrated that the levels of inorganic phosphate and phosphocreatine are significantly more sensitive to ischemic insult than are the levels of adenosine triphosphate. 19,20 The ratio of inorganic phosphate to phosphocreatine is directly associated With cellular phosphorylation potential. ${ }^{19,20}$ The efficacies of RC and AC in sustaining myocardial energy metabolism were therefore evaluated in this study by measuring the levels of inorganic phosphate and phosphocreatine.

\section{Material and Methods}

Animal Preparations

All animals received humane care in compliance With the Guide to the Care and Use of Experimental Animals formulated by the
Canadian Council on Animal Care. The protocols used in this study were approved by the Animal Care Committee of National Research Council of Canada.

Isolated rat hearts were obtained from 250- to 350-g male rats of the Sprague-Dawley strain. Animals were anesthetized With an intraperitoneal injection of sodium pentobarbital $(65 \mathrm{mg} / \mathrm{kg})$ and sodium heparin $(0.1 \mathrm{~mL})$. The abdominal cavity was opened by making a transverse incision With scissors. The diaphragm was transected, and lateral incisions were made along both sides of the rib cage. The hearts were rapidly excised and placed in cold cardioplegic solution.

Standard procedures for preparing isolated pig hearts have been described. ${ }^{21}$ The following is an outline of a few key procedures used in this study. Domestic pigs weighing 40 to $50 \mathrm{~kg}$ were sedated With an intramuscular injection of atropine $(0.5 \mathrm{mg} / \mathrm{kg}$ body weight), diazepam ( $0.4 \mathrm{mg} / \mathrm{kg}$ body weight), and ketamine $(20 \mathrm{mg} / \mathrm{kg}$ body weight). Anesthesia was maintained With $1.5 \%$ to $2.0 \%$ isoflurane in a mixture of oxygen and medical air. A sternotomy was performed, and the great arteries and veins were dissected and clamped. Heparinized cold (approximately $4^{\circ} \mathrm{C}$ ) cardioplegic solution was delivered into the aortic root to arrest the hearts. After removal, the pig hearts were immersed into the cold cardioplegic solution for instrument implantation.

Both the aorta and the left anterior descending coronary artery (LAD) were cannulated for AC. The anterior ascending cardiac vein $(\mathrm{AACV})$ and coronary sinus were cannulated for $\mathrm{RC}$. The $\mathrm{LAD}$ and the AACV were ligated proximally to prevent leakage of cardioplegic solution. The coronary sinus was cannulated With a $15 \mathrm{~F}$ retrograde cannula (DLP Inc, Grand Rapids, Mich) With a manually inflated balloon at the tip positioned approximately $1 \mathrm{~cm}$ into the coronary sinus. We did not place a purse-string suture around the coronary sinus.

\section{Protocols}

Protocol 1: Assessment of Capillary Flows of Retrograde and Antegrade Cardioplegia After removal from animals, 4 rat hearts were cannulated through the aorta and another 4 were cannulated through the coronary sinus. All hearts were then perfused With 4 $\mathrm{mL}$ of a mixture of autogenous blood and Krebs-Henseleit solution containing $6 \times 10^{6}$ colored microspheres With a diameter of 3.5 $\mu \mathrm{m}$. The colored microspheres were expected to flow freely through the capillaries because of their relatively small diameter. Infusion rates were similar $(1 \mathrm{~mL} / \mathrm{min})$ for $\mathrm{RC}$ and AC. After infusion of microspheres, the hearts were not subjected to any further perfusion but were dissected immediately into 2-mm thick blocks along the base-apex axis and frozen in liquid nitrogen. The tissue blocks were then cut into $16-\mu \mathrm{m}$ thick sections. The tissue sections underwent a two-step histochemical procedure to distinguish the arteriolar and venular portions of the capillaries. The endothelia in the arteriolar portion of the capillaries contain alkaline phosphatase and are stained blue. ${ }^{11,12}$ The endothelia in the venular portion have dipeptidyl peptidase IV and are stained red. ${ }^{11,12}$ With this technique, the capillaries can readily be separated into two anatomic portions. The numbers of colored microspheres located in the two portions of the capillaries were then determined under a light microscope. Rat hearts were used in this part of the study because they have relatively higher concentrations of the two enzymes than do pig hearts. ${ }^{11,12,22}$ It should be 
mentioned that the absolute value of the capillary flow could not be obtained With the approach used in this study. The capillary flows of $\mathrm{RC}$ and $\mathrm{AC}$ were assessed in a relative manner according to the numbers of colored microspheres found in the capillaries.

Protocol 2: Assessment of Regional Myocardial Perfusions of Retrograde and Antegrade Cardioplegia Six isolated pig hearts were perfused With a mixture of autogenous blood and KrebsHenseleit solution With 16-mmol/L potassium chloride and 16$\mathrm{mmol} / \mathrm{L}$ magnesium chloride. AC was performed through the LAD and the aorta simultaneously. Flow rate at the LAD was 30 $\mathrm{mL} / \mathrm{min}$. The head pressure at the aorta and LAD was set at the same level to minimize collateral flow between the LAD and non-LAD vessels. RC was carried out through the AACV and the coronary sinus simultaneously. Cardioplegic flow at the AACV was set at $30 \mathrm{~mL} / \mathrm{min}$ to keep it at the same level as that at the LAD during AC. Perfusion pressures at the AACV and the coronary sinus were kept similar to diminish collateral flow between the AACV and adjacent cardiac veins. Perfusion pressures at the aorta, the LAD, the coronary sinus, and the AACV were monitored With a Polygraph Data Recording System (model 79E; Grass Instrument Co, Quincy, Mass) equipped With a pressure transducer connected distally to the perfusion catheters. Pressure calibration was performed before each experiment to ensure that there were no differences between pressure readings and actual perfusion pressure.

One milliliter of a blood-pool magnetic resonance (MR) contrast agent (gadolinium diethylenetriamine pentaacetic acid polylysine) was injected into the AACV and the LAD catheter during $\mathrm{RC}$ and $\mathrm{AC}$, respectively. Its first pass was monitored With T2* MR imaging. Perfusion medium containing the contrast agent was discarded and not allowed to recirculate into the perfusion system. Once the contrast agent was completely washed out, $1 \mathrm{~mL}$ of an extracellular type MR contrast agent (gadolinium diethylenetriamine pentaacetic acid) was given at the same sites. Its first pass was followed With MR imaging. It has been shown that T2* MR imaging in combination With a blood-pool contrast agent is more sensitive to microvascular perfusion, whereas T1 MR imaging in conjunction With an extracellular MR contrast agent is more sensitive to diffusion of the perfusion medium. ${ }^{23}$ Use of these two imaging techniques together is expected to provide a more complete picture of myocardial perfusion of RC and AC than use of either alone.

MR imaging was performed in a 7-T, 40-cm horizontal-bore magnet equipped With a Biospec spectrometer (Bruker Inc, Karlsruhe, Germany) and a Helmholtz probe surrounding the whole heart. Each image covered a $12 \times 12-\mathrm{cm}^{2}$ field of view With a $128 \times 128$ matrix, leading to a pixel size of $0.93 \times 0.93$ $\mathrm{mm}^{2}$. All images were acquired from a $3-\mathrm{mm}$ slice parallel to the short cardiac axis.

After MR imaging studies, the pig hearts were perfused retrogradely through the coronary sinus and the AACV simultaneously at a perfusion pressure of $40 \mathrm{~mm} \mathrm{Hg}$. Effluents at the coronary arteries and both ventricles were measured to determine the draining percentage of the outlets and to estimate the capillary flow of $\mathrm{RC}$, because most of the effluent collected at the coronary arteries had flowed through the capillaries. The arterial catheters were open to air during $\mathrm{RC}$ and positioned at the same level of the midpoint of the heart. In this way arterial catheters would not impose significant resistance to the efflux of RC.

Protocol 3: Assessment of Abilities of Retrograde and Antegrade Cardioplegia to Sustain Myocardial Energy Homeostasis Six isolated pig hearts were perfused With the hyperkalemic mixture of pig blood and Krebs-Henseleit solution used in protocol 2. The hearts were cannulated in the same way as described in protocol 2. RC was performed through the AACV and the coronary sinus simultaneously. Perfusion pressures at the AACV and the coronary sinus during $\mathrm{RC}$ were the same. AC was carried out through the LAD and the aorta simultaneously. Likewise, head pressures at the LAD and the aorta during $\mathrm{AC}$ were comparable. Four flow rates were used at the AACV during RC and at the LAD during AC. These flow rates were $20 \mathrm{~mL} / \mathrm{min}, 10 \mathrm{~mL} / \mathrm{min}, 5$ $\mathrm{mL} / \mathrm{min}$, and $3 \mathrm{~mL} / \mathrm{min}$. Each flow rate was carried out for 20 minutes to allow its effects on myocardial energy metabolism to be assessed. RC and AC were alternated in each heart. Between each 20-minute period at one of the four flow rates, a 5-minute highflow perfusion was carried out through the aorta and the LAD at a total flow rate of $300 \mathrm{~mL} / \mathrm{min}$ to resuscitate the heart. Complete recovery of myocardial high-energy phosphates was a prerequisite for subsequent RC and AC. Changes in myocardial high-energy phosphates in the LAD region were monitored with localized phosphorus $31 \mathrm{MR}$ spectroscopy.

Localized phosphorus $31 \mathrm{MR}$ spectroscopy was performed in the same magnet used for the MR imaging studies. A $1.0-\mathrm{cm}$ diameter surface coil was positioned over the LAD region of myocardium. Each phosphorus 31 spectrum was averaged across a 2-minute sample period. The observed phosphorus compounds included inorganic phosphate, phosphocreatine, and three peaks of adenosine triphosphate ( $\alpha, \beta$, and $\gamma$ peaks). Peak areas were used to assess metabolite levels.

\section{Data Analysis}

Statistical analyses were performed with STATISTICA software (StatSoft Inc, Tulsa, Okla). All numeric results are expressed as the mean $\pm \mathrm{SD}$ of the mean. Significant decreases in phosphocreatine and increases in inorganic phosphate during each 20-minute RC and AC were determined with 1-way analysis of variance. The Student $t$ test was used to compare the numbers of total colored microspheres in the capillaries between the hearts subjected to RC and to AC. This test was also used to compare the distributions of colored microspheres in the arteriolar and venular portions of the capillaries between the two groups of hearts.

To compare the regional myocardial perfusions of $\mathrm{RC}$ and $\mathrm{AC}$, we measured the overall amount of the intercorrelation between each signal-time course of $\mathrm{T} 2 *$ and $\mathrm{T} 1$ images obtained from the left ventricular wall during $\mathrm{RC}$ and $\mathrm{AC}$. The overall amount of the intercorrelation was determined by the method of Gleason and Staelin. ${ }^{24}$ It ranged from 0 (when no signal-time courses were associated with any other) to 1 (when they were perfectly correlated with each other).

\section{Results}

As shown in Figure 1, RC delivered significantly fewer colored microspheres $\left(15 \pm 1 \mathrm{microspheres} / \mathrm{mm}^{2}\right)$ to the capillaries than did AC $\left(29 \pm 2\right.$ microspheres $\left./ \mathrm{mm}^{2}\right)$. This indicates that RC provided significantly less capillary flow 


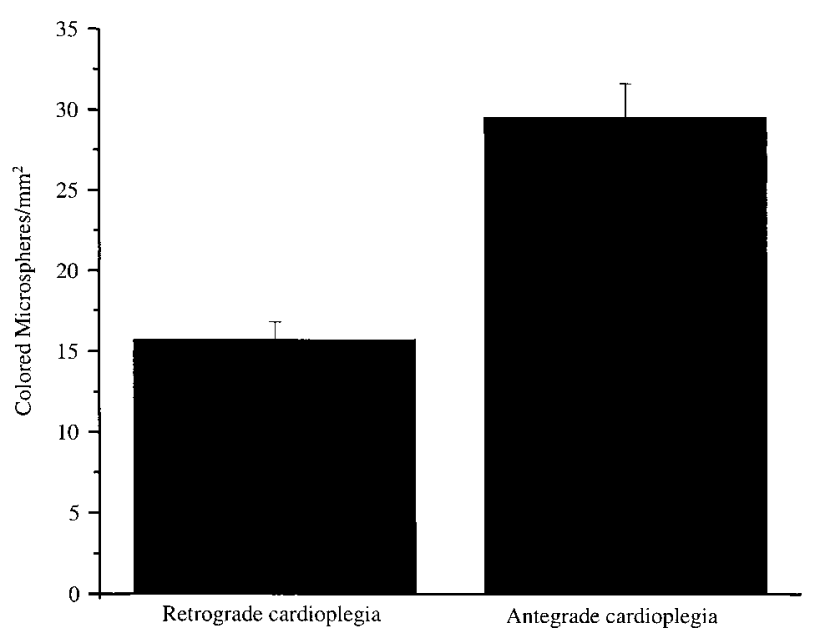

Figure 1. Numbers of colored microspheres found in capillaries of hearts subjected to RC and to AC. Bars represent mean; error bars represent SD.

than did $\mathrm{AC}$ at the same cardioplegic flow. In addition, the infusion rate of $1 \mathrm{~mL} / \mathrm{min}$ gave rise to perfusion pressures of $35 \pm 4 \mathrm{~mm} \mathrm{Hg}$ and $12 \pm 2 \mathrm{~mm} \mathrm{Hg}$ for $\mathrm{RC}$ and $\mathrm{AC}$, respectively. Furthermore, it was noted that colored microspheres were evenly distributed between the arteriolar $(51.3 \% \pm 2.4 \%$ of total colored microspheres) and venular portions $(48.6 \% \pm 2.4 \%)$ of the capillaries when they were given with AC (Figure 2). In contrast, when colored microspheres were given with RC, $80.2 \% \pm 3.2 \%$ were located in the venular portions of the capillaries. Only $19.1 \% \pm 3.4 \%$ of the colored microspheres were found in the arteriolar portion of the capillaries. This indicates that some of the RC solution reaching the capillaries did not flow through the capillaries.

The percentages of effluents collected at various sites of the pig hearts during $\mathrm{RC}$ and their $\mathrm{Po}_{2}$ values are shown in Figure 3. As expected, most $(58.7 \% \pm 6.8 \%)$ of the RC solution drained directly into the right ventricle. The left ventricle collected only $6.8 \% \pm 2.4 \%$ of RC solution. The LAD, the left circumflex coronary artery, and the right coronary artery drained $17.8 \% \pm 4.2 \%, 15.2 \% \pm 2.6 \%$, and $3.8 \% \pm 4.8 \%$ of RC solution, respectively. As such, approximately $36 \%$ of RC solution drained from the three coronary arteries under our experimental conditions. $\mathrm{Po}_{2}$ values of the effluents recovered from the right and left ventricles were $198 \pm 123 \mathrm{~mm} \mathrm{Hg}$ and $109 \pm 127 \mathrm{~mm} \mathrm{Hg}$, respectively. The levels of $\mathrm{Po}_{2}$ were considerably higher than those measured from the effluents recovered at the LAD $(41 \pm 15 \mathrm{~mm} \mathrm{Hg})$, the left circumflex coronary artery $(41 \pm 21 \mathrm{~mm} \mathrm{Hg})$, and the right coronary artery $(28 \pm 5$ $\mathrm{mm} \mathrm{Hg}$ ).

Representative signal-time courses of $\mathrm{T} 2 *$ images obtained from the anterior wall of the left ventricle during RC

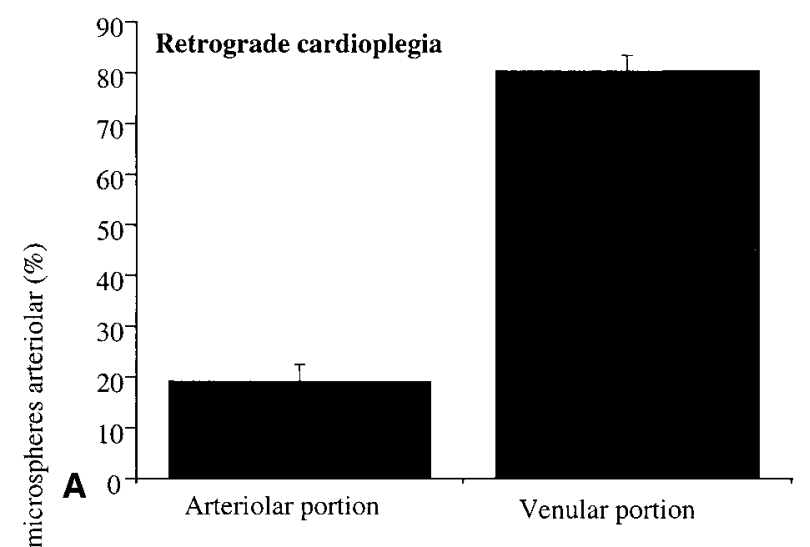

Figure 2. Distributions of colored microspheres in arteriolar and venular portions of capillaries in $\mathrm{RC}(\mathrm{A})$ and $\mathrm{AC}(\mathrm{B})$. In hearts subjected to $A C$, percentages of colored microspheres in both arteriolar and venular portions of capillaries were not significantly different $(P>.05)$. Difference in percentage of colored microspheres in portions of capillaries was statistically significant for RC. Bars represent mean; error bars represent SD.

and $\mathrm{AC}$ are shown in Figure $4, A$ and $B$, respectively. The profiles of the signal-time courses acquired during RC differed significantly from each other, whereas those obtained during $\mathrm{AC}$ were quite similar. The intercorrelation of the $\mathrm{T} 2 *$ signal-time courses obtained during $\mathrm{RC}(0.515 \pm 0.15)$ was significantly $(P=.0016)$ weaker than that of the signal-time courses acquired during AC (0.839 \pm 0.07$)$. This suggests that microvascular perfusion of RC varied significantly among the various small areas of the myocardium relative to that of AC. Signal-time courses of T1 images obtained during $\mathrm{RC}$ and $\mathrm{AC}$ are shown in Figure 5, $A$ and $B$, respectively. Likewise, the overall intercorrelation of the signal-time courses obtained during RC (0.489 \pm 0.09) was significantly weaker than that of those during AC $(0.86 \pm 0.12)$. This finding demonstrates that diffusion of RC solution into the interstitial space was also uneven among the various small areas of the myocardium. In addition, a flow rate of $30 \mathrm{~mL} / \mathrm{min}$ at the LAD during AC gave 

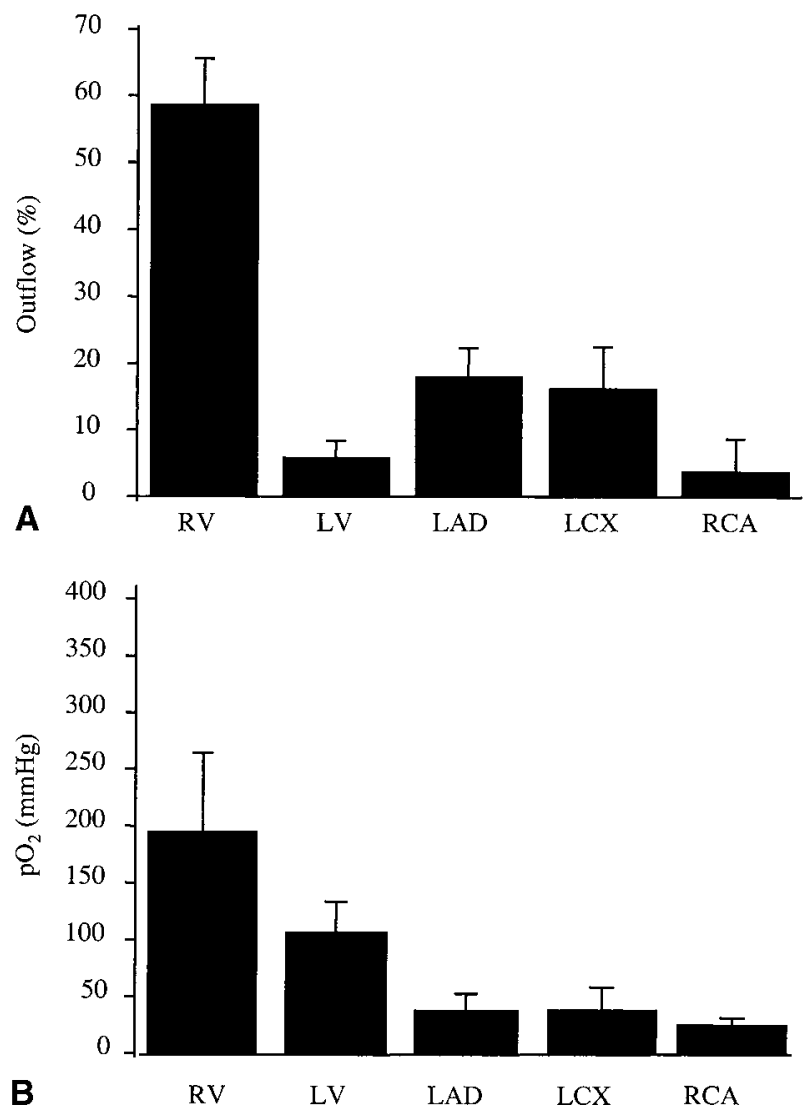

Figure 3. A, Drainage percentages of $\mathrm{RC}$ solution at various sites. $\mathrm{B}, \mathrm{Po}_{2}$ values measured at various locations during $\mathrm{RC}$. Bars represent mean; error bars represent SD. $R V$, Right ventricle; $L V$, left ventricle; $L C X$, left circumflex coronary artery; $R C A$, right coronary artery.

rise to a perfusion pressure of $29 \pm 4 \mathrm{~mm} \mathrm{Hg}$, whereas the same flow rate at the AACV was associated with a significantly higher perfusion pressure at the venous end (55 \pm 11 $\mathrm{mm} \mathrm{Hg}$ ).

The levels of myocardial inorganic phosphate and phosphocreatine measured during $\mathrm{RC}$ and $\mathrm{AC}$ at a flow rate of 20 $\mathrm{mL} / \mathrm{min}$ are shown in Figure 6. No significant changes in the energy metabolites were observed during 20 minutes of RC or $\mathrm{AC}$ at this flow rate, indicating that both cardioplegic techniques at this level of the flow rate were able to sustain normal myocardial energy metabolism. At a flow rate of 10 $\mathrm{mL} / \mathrm{min}, \mathrm{RC}$ resulted in a gradual and significant decrease in phosphocreatine and an increase in inorganic phosphate, which indicates myocardial ischemia (Figure 7). At the same cardioplegic flow, AC was still able to maintain normal levels of myocardial energy metabolites (Figure 7). AC resulted in an apparent decline in phosphocreatine and increase in inorganic phosphate at a flow rate of $3 \mathrm{~mL} / \mathrm{min}$ (Figure 8). No significant changes were observed in aden-
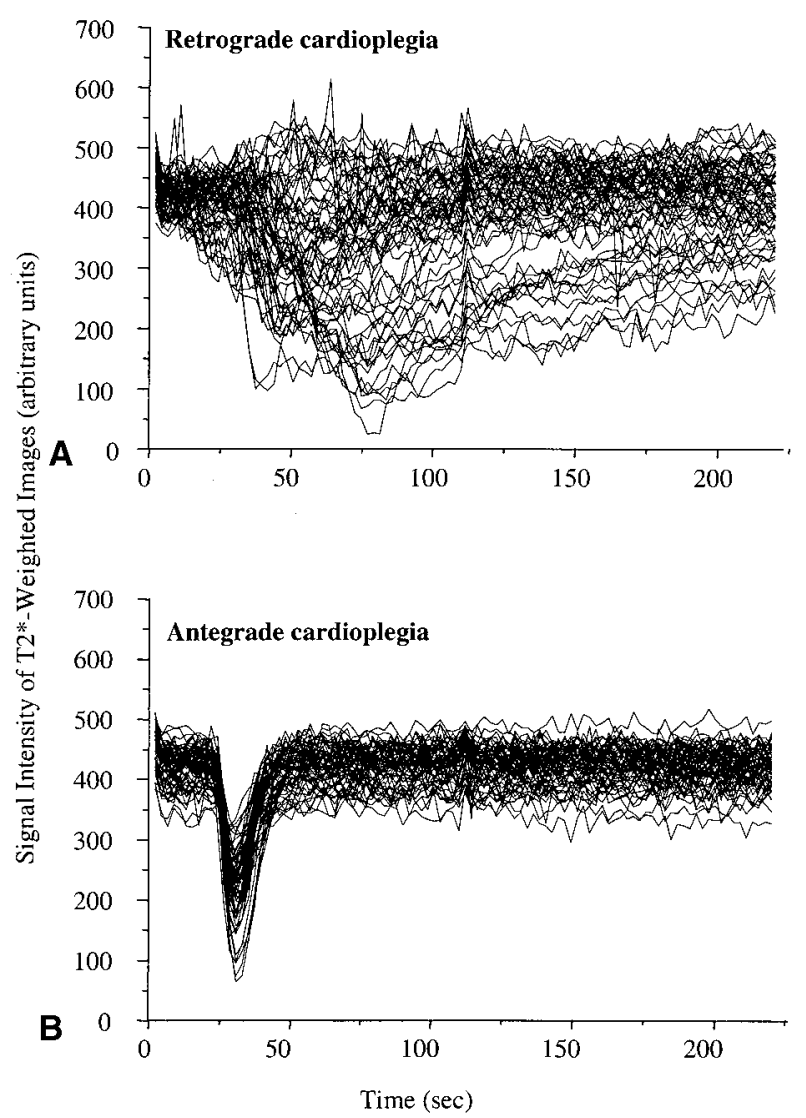

Figure 4. Signal-time courses of $\mathrm{T}^{*}$ images obtained during RC (A) and AC (B). It is clear that injection of gadolinium diethylenetriamine pentaacetic acid polylysine during $A C$ resulted in similar changes in $\mathrm{T}^{*}$ signal intensities, whereas contrast-induced T2* changes during RC varied significantly among pixels of myocardium.

osine triphosphate level during 20 minutes of RC (at a flow $10 \mathrm{~mL} / \mathrm{min}$ ) and $\mathrm{AC}$ (at a flow of $3 \mathrm{~mL} / \mathrm{min}$, data not shown). Perfusion pressures at the arterial end during AC were $3.7 \pm 2.1 \mathrm{~mm} \mathrm{Hg}, 5.4 \pm 3.2 \mathrm{~mm} \mathrm{Hg}, 12.3 \pm 5.1 \mathrm{~mm}$ $\mathrm{Hg}$, and $20.4 \pm 3.5 \mathrm{~mm} \mathrm{Hg}$ at flow rates of $3 \mathrm{~mL} / \mathrm{min}, 5$ $\mathrm{mL} / \mathrm{min}, 10 \mathrm{~mL} / \mathrm{min}$, and $20 \mathrm{~mL} / \mathrm{min}$, respectively. As expected, these flow rates gave rise to much higher perfusion pressures at the venous end during $\mathrm{RC}$. The perfusion pressures were $15.2 \pm 8.2 \mathrm{~mm} \mathrm{Hg}, 23.3 \pm 10 \mathrm{~mm} \mathrm{Hg}$, and $41.5 \pm 12.4 \mathrm{~mm} \mathrm{Hg}$ at flow rates of $5 \mathrm{~mL} / \mathrm{min}, 10 \mathrm{~mL} / \mathrm{min}$, and $20 \mathrm{~mL} / \mathrm{min}$, respectively. Obvious ischemic changes were observed during $\mathrm{RC}$ at a flow rate of $10 \mathrm{~mL} / \mathrm{min}$. Therefore, phosphorus $31 \mathrm{MR}$ spectroscopy was not performed at lower flow rates ( 3 and $5 \mathrm{~mL} / \mathrm{min}$ ) during $\mathrm{RC}$.

\section{Discussion}

$\mathrm{RC}$ has been widely used for heart protection during cardiac surgery because of its ability to deliver blood cardioplegia 


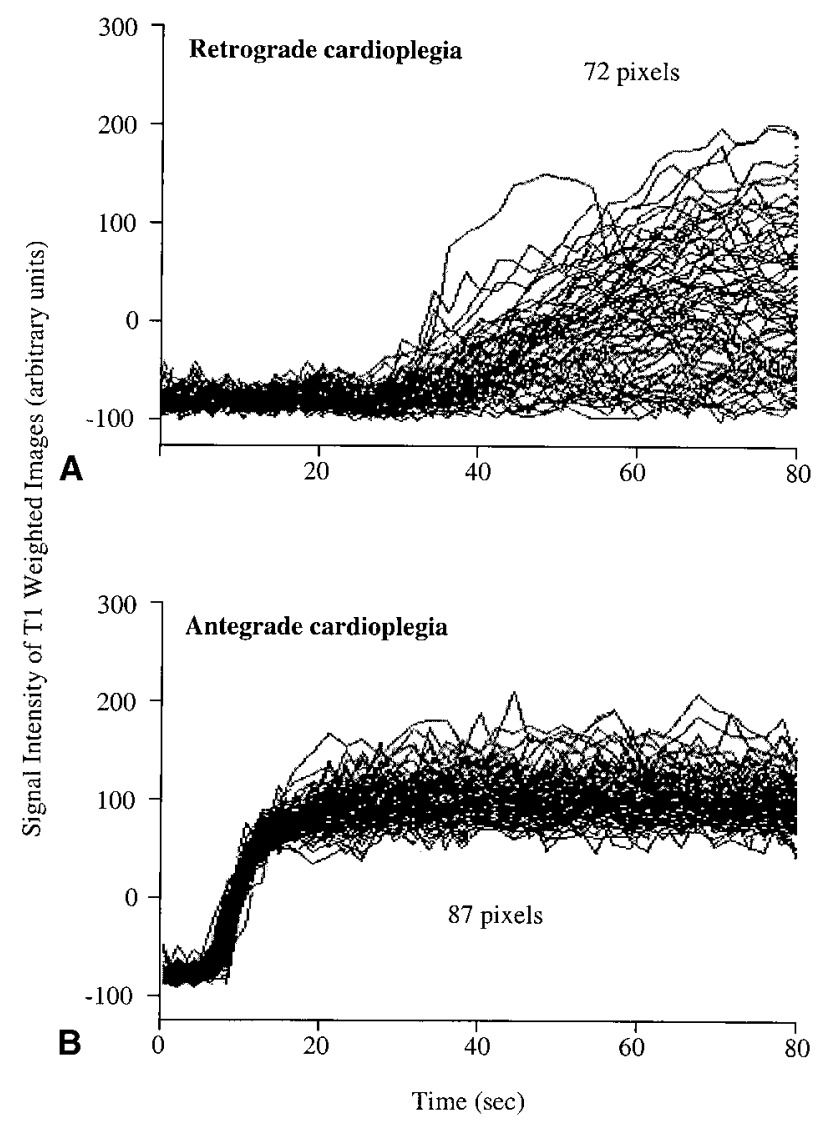

Figure 5. Signal-time courses of T1 images obtained during RC (A) and $A C(B)$.

to the jeopardized myocardium and other advantages. However, its efficacy in myocardial perfusion has not been adequately demonstrated. This study compared the capillary flows, regional myocardial perfusions, and abilities to sustain myocardial energy homeostasis of RC and $\mathrm{AC}$ at the same cardioplegic flows. We found that RC provided significantly less capillary flow than did AC. Its regional myocardial perfusion varied significantly at a microscopic level. As a result, a higher blood flow was needed during $\mathrm{RC}$ than during AC to sustain normal myocardial energy metabolism. The results demonstrate that RC is associated with less effective myocardial perfusion than is AC.

In the first part of the study, we found that RC delivered significantly fewer colored microspheres $(15.7 \pm 1.1 \mathrm{mi}-$ crospheres $\left./ \mathrm{mm}^{2}\right)$ to the capillaries than $\operatorname{did} \mathrm{AC}(29.5 \pm 2.1$ microspheres $/ \mathrm{mm}^{2}$ ). According to the number of the total colored microspheres, apparent capillary flow of RC was estimated to be $53 \%(15.7 / 29.5)$ of that of AC. This suggests that the capillary flow of RC is significantly lower than that of $\mathrm{AC}$ at the same cardioplegic flow. Blood drainage into the ventricular chambers through the Thebesian vessels is believed to be the principal cause of the lower capillary flow

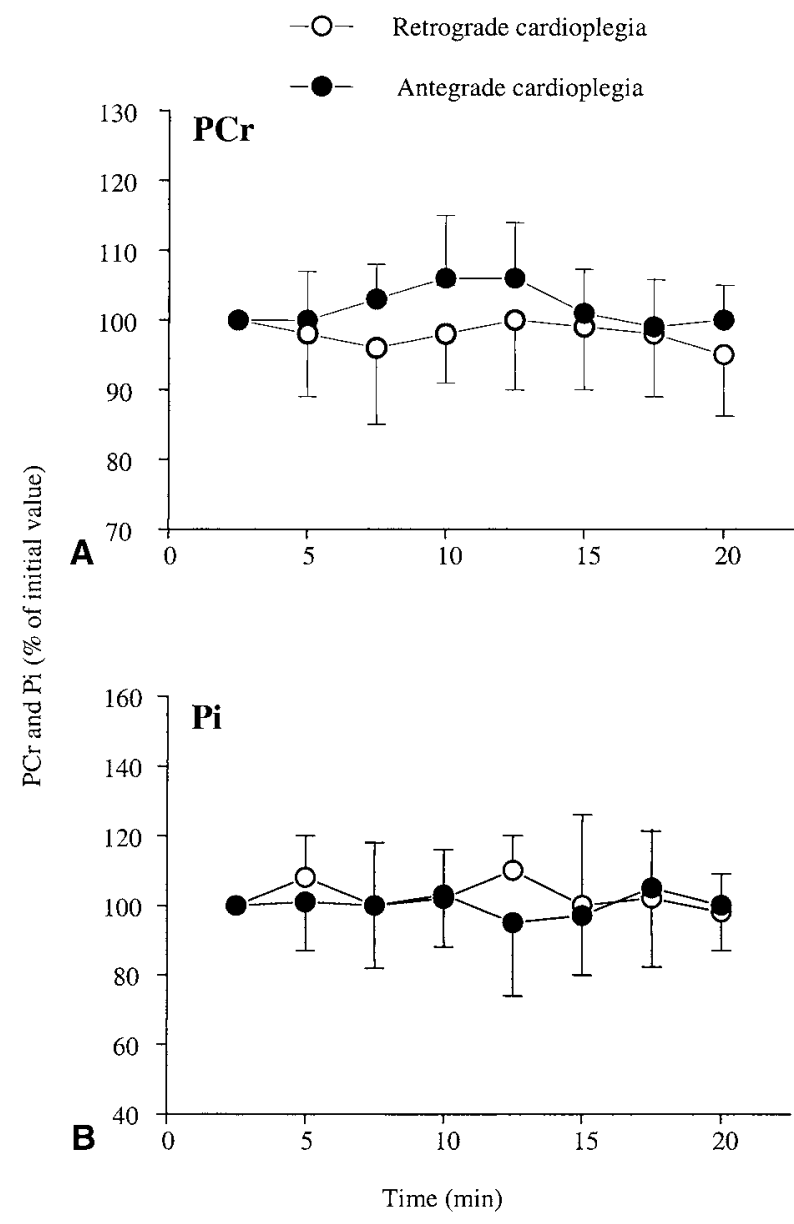

Figure 6. Time courses of phosphocreatine $(P C r, A)$ and inorganic phosphate ( $\mathrm{Pi}, \mathrm{B})$ obtained during $\mathrm{RC}$ (open circles) and AC (closed circles) at cardioplegic flow rate of $20 \mathrm{~mL} / \mathrm{min}$. There were no significant changes in energy metabolites throughout measurements. Data points represent mean; error bars represent SD.

of RC. Moreover, we also found that distribution of colored microspheres in the venular and arteriolar portions of the capillaries was highly uneven during RC. Of $15.7 \pm 1.3$ microspheres $/ \mathrm{mm}^{2}$ in the capillaries, only $3 \pm 0.4$ microspheres $/ \mathrm{mm}^{2}$ were found in the arteriolar portion. Most of the colored microspheres remained in the venular portions of the capillaries. In contrast, colored microspheres were distributed more evenly in the arteriolar (15.2 \pm 1.8$)$ and venular $(14.3 \pm 1.0)$ portions of the capillaries during AC. The results indicate that some of the RC solution was diverted from the capillaries at the venular end, presumably through the Thebesian vessels. If only colored microspheres found in the arteriolar portions of the capillaries $(3.0 \pm 0.4$ microspheres $/ \mathrm{mm}^{2}$ ) during $\mathrm{RC}$ were considered to represent the capillary flow of $\mathrm{RC}$, and those found in the venular portions $(14.3 \pm 1.0)$ during $\mathrm{AC}$ reflect the capillary flow of 


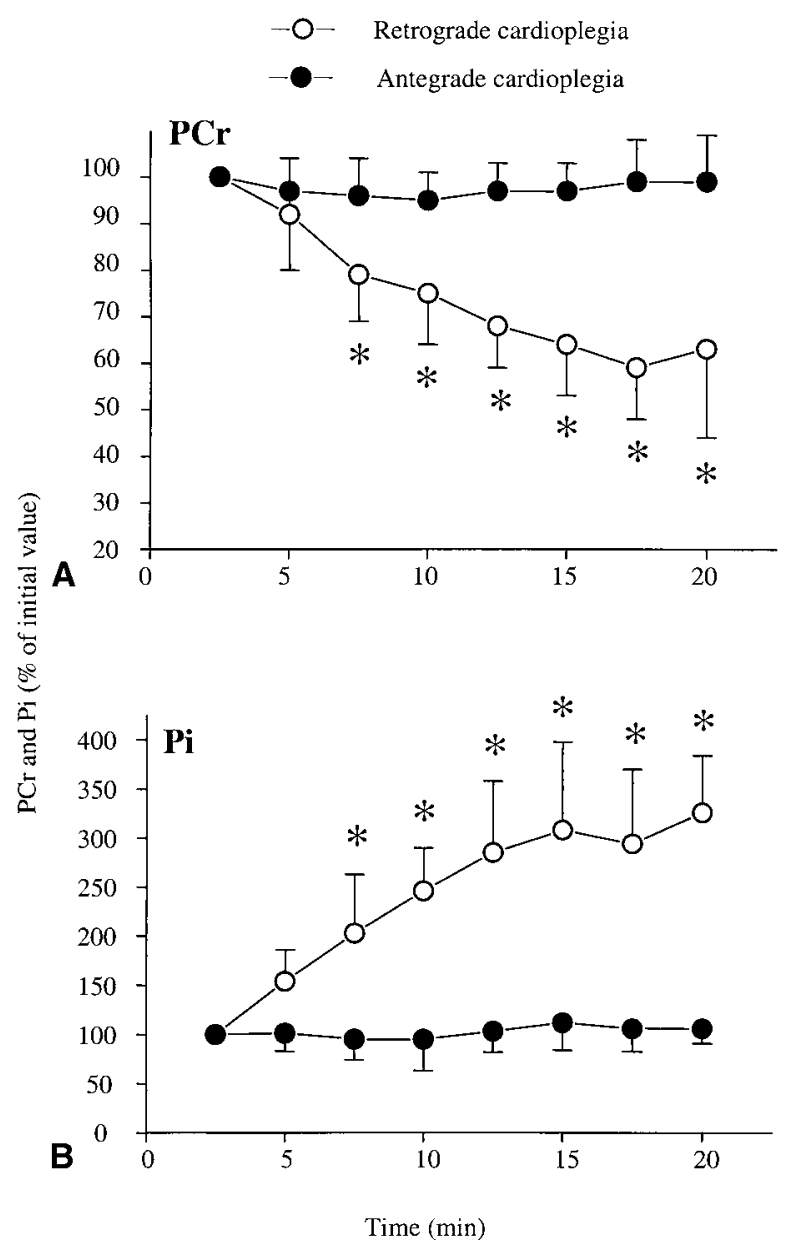

Figure 7. Time courses of phosphocreatine $(P C r, A)$ and inorganic phosphate (Pi, B) obtained during $\mathrm{RC}$ (open circles) and $\mathrm{AC}$ (closed circles) at cardioplegic flow rate of $10 \mathrm{~mL} / \mathrm{min}$. RC resulted in gradual and significant decrease in phosphocreatine and increase in inorganic phosphate. Data points represent mean; error bars represent SD. Asterisk indicates significant difference from control value.

AC, the capillary flow of RC was estimated to be approximately $20 \%$ of that of AC. This level of capillary flow of RC is considerably smaller than that (53\%) calculated according to the total colored microspheres in the capillaries. This suggests that use of total colored microspheres in the capillaries may overestimate the capillary flow of RC.

As shown in Figure 3, more than $60 \%$ of the RC solution was diverted from the capillaries and drained directly into the ventricular chambers. Only approximately $36 \%$ of the $\mathrm{RC}$ solution was collected at the coronary arteries. If the effluents recovered at the coronary arteries flowed through the capillaries, the ratio of capillary flow to total flow was approximately $36 \%$. If capillary flow ratio (capillary flow/ total flow) of AC was near $100 \%$, the capillary flow of RC

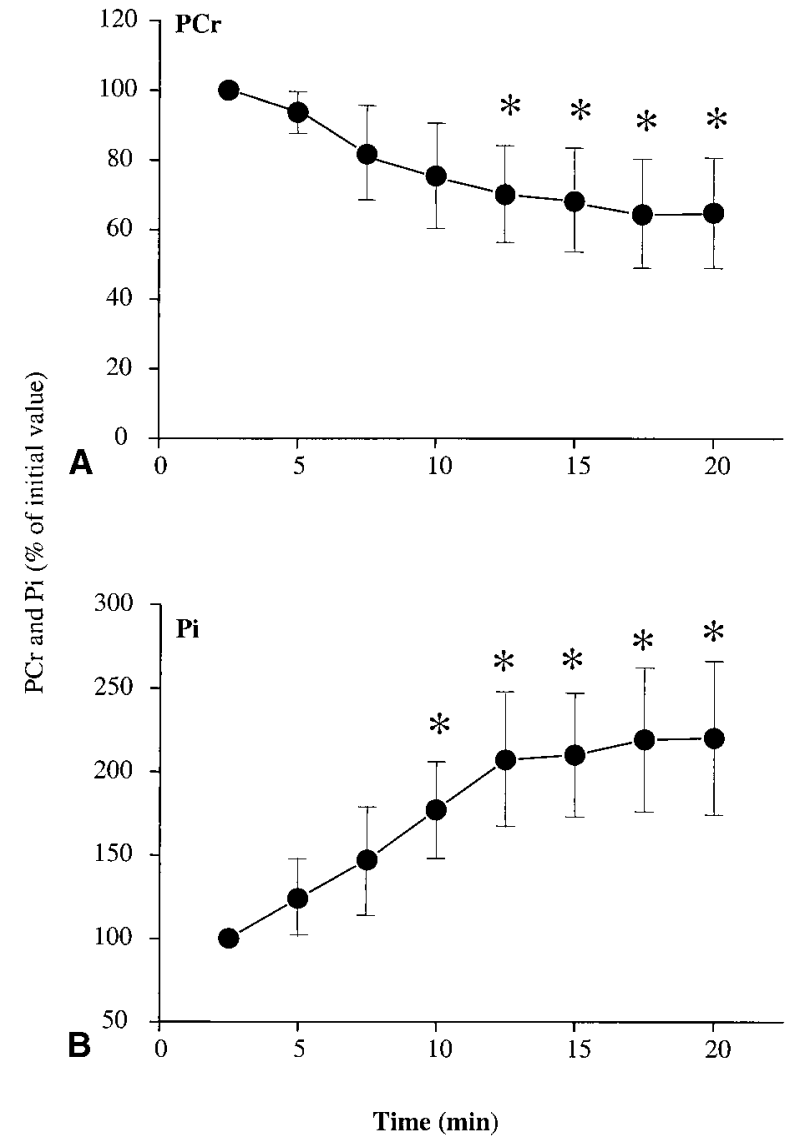

Figure 8. Time courses of phosphocreatine $(P C r, A)$ and inorganic phosphate (Pi, B) obtained during $A C$ at flow rate of $3 \mathrm{~mL} / \mathrm{min}$. Data points represent mean; error bars represent SD. Asterisk indicates significant difference from control value.

was about $36 \%$ of that of AC in the pig hearts. This ratio is significantly higher than that (approximately 20\%) calculated according to colored microspheres found in the arteriolar and venular portions of the capillaries for $\mathrm{RC}$ and $\mathrm{AC}$, respectively. The discrepancy in capillary flow of RC may be related to the facts that different animals and approaches were used in part 1 of the study from those in parts 2 and 3. On the other hand, this ratio (36\%) is significantly lower than that $(53 \%)$ obtained by measuring the total colored microspheres in the capillaries, which also indicates that the total number of colored microspheres overestimates the capillary flow.

MR imaging has been used extensively for assessment of tissue perfusion because of its noninvasive and nondestructive properties. It has been shown that $\mathrm{T} 2 *$ imaging is sensitive to the heterogeneity of the local magnetic field. ${ }^{25,26}$ When a blood-pool contrast agent is flushing into the capillaries, the microscopically heterogeneous distribution of the contrast agent creates local magnetic gradients at the 
boundaries of the capillaries. These local magnetic gradients result in a rapid decrease in $\mathrm{T} 2 *$ signal intensities. ${ }^{26} \mathrm{As}$ the contrast agent is washed out of the capillaries, the signal intensities recover gradually. Obviously the rates of $\mathrm{T} 2 *$ signal decline and recovery are dependent directly on myocardial perfusion at the microvascular level. A region with a higher blood flow would show a more rapid decline and recovery in $\mathrm{T} 2 *$ signal intensities relative to a region with a lower blood flow. As mentioned previously, the intercorrelation of the $\mathrm{T} 2 *$ signal-time courses obtained during $\mathrm{RC}$ was significantly weaker than that obtained during AC. This suggests that blood flow rate varied significantly among the various small areas of the myocardium. In contrast, $\mathrm{T} 2 *$ signal-time courses obtained during $\mathrm{AC}$ appeared more uniform, suggesting that microvascular perfusion with $\mathrm{AC}$ was more similar between each pixel of the myocardium in the same region.

In the third part of the study, we compared the abilities of $\mathrm{RC}$ and $\mathrm{AC}$ to sustain normal myocardial energy metabolism. RC could not maintain normal energy level at a flow of $10 \mathrm{~mL} / \mathrm{min}$, whereas $\mathrm{AC}$ did not result in any ischemic changes until its flow rate was reduced to $3 \mathrm{~mL} / \mathrm{min}$. This finding demonstrates that $\mathrm{RC}$ has a lower perfusion efficacy than does AC.

It has been shown that the rate of oxygen consumption in a pig heart under normothermic conditions is about 6.7 $\mu \mathrm{L} /(\mathrm{g} \times \mathrm{min}){ }^{27,28}$ On the basis of the hemoglobin concentration $(5.1 \pm 0.1 \mathrm{~g} / \mathrm{dL})$ and the $\mathrm{Po}_{2}(545 \pm 27 \mathrm{~mm} \mathrm{Hg})$ of our perfusion medium, the LAD region of myocardium (myocardium supported by the LAD and presumably drained by the AACV) is expected to need approximately $6.9 \mathrm{~mL}$ blood cardioplegic solution to sustain normal myocardial energy metabolism. At a flow rate of $10 \mathrm{~mL} / \mathrm{min}, \mathrm{RC}$ is expected to provide approximately 2 to $3.6 \mathrm{~mL}$ of capillary flow according to the ratio of capillary flow to total flow estimated in this study. As such, RC was unable to maintain normal energy levels at this flow rate. Because most antegradely delivered blood cardioplegia flows through the capillaries, AC was able to sustain normal myocardial energy metabolism at this flow rate $(10 \mathrm{~mL} / \mathrm{min})$. There were no obvious ischemic changes during $\mathrm{AC}$ at a flow rate of 5 $\mathrm{mL} / \mathrm{min}$, which may have been due to the small difference between energy supply and demand. Significant ischemic changes were found during $\mathrm{AC}$ when its flow rate was reduced to $3 \mathrm{~mL} / \mathrm{min}$. The large difference in threshold flow (the flow starting to cause ischemic changes) between $\mathrm{RC}$ $(10 \mathrm{~mL} / \mathrm{min})$ and $\mathrm{AC}(3 \mathrm{~mL} / \mathrm{min})$ indicates that the efficacy of myocardial perfusion of $\mathrm{RC}$ is lower than that of $\mathrm{AC}$. The low capillary flow ratio and variation in microvascular perfusion of $\mathrm{RC}$ were believed to be the principal cause for the low perfusion efficacy. In addition, if the volume of the myocardium drained by the AACV was significantly larger than that supported by the LAD, the difference in tissue volume might also be partially responsible for the disparity in the threshold flow. In 3 pig hearts, we injected gadolinium diethylenetriamine pentaacetic acid into the AACV during $\mathrm{RC}$ and into the LAD during $\mathrm{AC}$. We then measured the sizes of the contrast-enhanced areas of the myocardium on three slices. We found no significant difference between the sizes of LAD myocardium and AACV myocardium (data are not presented). This suggests that the difference in tissue volume was not the main cause of the disparity in the threshold cardioplegic flow between RC and AC.

It should be pointed out that the results of this study by no means indicate that $\mathrm{RC}$ is unable to sustain normal myocardial energy metabolism. As demonstrated in this study and our previous studies, ${ }^{29} \mathrm{RC}$ at an adequate perfusion pressure (approximately $40 \mathrm{~mm} \mathrm{Hg}$ ) is sufficient to prevent ischemic injury. In addition, the capillary flow and myocardial perfusion of $\mathrm{RC}$ and $\mathrm{AC}$ were assessed in this study with rat and pig hearts, respectively. The anatomic structures of the coronary microvasculature may differ somewhat between these animals. The differences may be partially responsible for the disparity in the capillary flow observed in the parts 1 and 2 of the study. Moreover, it has been shown that Thebesian flow of RC may have a certain degree of nutritional function. ${ }^{30}$ This suggests that the actual nutritional flow ratio (flow having nutritional function as a proportion of total flow) of RC may be higher than the capillary flow ratio estimated in this study. Nevertheless, low capillary flow and uneven microvascular perfusion may lead to ischemia if $\mathrm{RC}$ is used for a prolonged period or at an inadequate perfusion pressure. The combination of $\mathrm{RC}$ with AC may be the optimal approach for myocardial preservation.

In summary, RC provides significantly less capillary flow than does $\mathrm{AC}$ at the same flow rate. Its regional myocardial perfusion varies significantly among the various small areas of the myocardium. As a result, efficacy of RC to sustain normal myocardial energy homeostasis is lower than that of AC.

We thank Mr Allan Turner, Mrs Lori Gregorash, Mrs Rachelle Mariash, and Mrs Shelley Germscheid for their technical assistance with animal preparation and heart perfusion.

\section{References}

1. Pratt FH. The nutrition of the heart through the vessels of Thebesian and the coronary veins. Am J Physiol. 1898;1:86-103.

2. Lillehei CW, DeWall RA, Gott VL, Varco RL. Direct vision correction of calcific aortic stenosis by means of pump-oxygenator and retrograde coronary sinus perfusion. Dis Chest. 1956;30:123-32.

3. Salerno TA, Christakis GT, Abel J, Houck J, Barrozo CA, Fremes SE, et al. Technique and pitfalls of retrograde continuous warm blood cardioplegia. Ann Thorac Surg. 1991;51:1023-5.

4. Smith GT, Geary GG, Blanchard W, McNamara JJ. Reduction in infarct size by synchronized selective coronary venous retroperfusion of arterialized blood. Am J Cardiol. 1981;48:1064-70.

5. Vaage J. Retrograde cardioplegia: when and how. A review. Scand J Thorac Cardiovasc Surg Suppl. 1993;41:59-66. 
6. Engleman RM. Retrograde continuous warm blood cardioplegia. Ann Thorac Surg. 1991;51:180-1.

7. Partington MT, Ascar C, Buckberg GD, Julia P, Kofsky ER, Bugyi H. Studies of retrograde cardioplegia. I. Capillary blood flow distribution to myocardial supplied by open and occluded arteries. $J$ Thorac Cardiovasc Surg. 1989;97:605-12.

8. Caldarone CA, Krukenkamp IB, Misare BD, Levitsky S. Perfusion deficits with retrograde warm blood cardioplegia. Ann Thorac Surg. 1994;57:403-6.

9. Cohen MV, Matsuki T, Downey JM. Pressure-flow characteristics and nutritional capacity of coronary veins in dogs. Am J Physiol 1988; 255(4 Pt 2):H834-46.

10. Ardehali A, Laks H, Drinkwater DC, Gates RN, Kaczer E. Ventricular effluent of retrograde cardioplegia in human hearts has traversed capillary beds. Ann Thorac Surg. 1995;60:78-83.

11. Batra S, Koyama T, Gao M, Horimoto M, Rakusan K. Microvascular geometry of the rat heart arteriolar and venular capillary regions. Jpn Heart J. 1992;33:817-27.

12. Batra S, Rakusan K, Campbell SE. Geometry of capillary networks in hypertrophied rat heart. Microsvasc Res. 1991;41:29-40.

13. Waller C, Hiller KH, Voll S, Haase A, Ertl G, Bauer WR. Myocardial perfusion imaging using a non-contrast agent MR imaging technique. Int J Cardiovasc Imaging. 2001;17:123-32.

14. Ohtsuka S, Suzuki S, Ishikawa K, Uchida W, Yatsu T, Yamaguchi I. Norepinephrine release is increased in the hibernating heart, studied in a chronic canine model of myocardial hibernation. J Cardiovasc Pharmacol 2000;36 Suppl 2:S35-41.

15. Aronson S, Lee BK, Zaroff JG, Wiencek BS, Walker R, Feinstein S, et al. Myocardial distribution of cardioplegic solution after retrograde delivery in patients undergoing cardiac surgical procedures. $J$ Thorac Cardiovasc Surg. 1993;105:214-21.

16. Haan C, Lazar HL, Bernard S, Rivers S, Zallnick J, Shemin RJ. Superiority of retrograde cardioplegia after acute coronary occlusion. Ann Thorac Surg. 1991;51:408-12.

17. Villanueva FS, Spotnitz WD, Glasheen WP, Watson DD, Jayaweera AR, Kaul S. New insights into the physiology of retrograde cardioplegia delivery. Am J Physiol. 1995;268(4 Pt 2):H1555-66.

18. Cohen MV, Matsuki T, Downey JM. Pressure-flow characteristics and nutritional capacity of coronary veins in dogs. Am J Physiol. 1988; 255(4 Pt 2):H834-46.
19. Opie LH. ATP synthesis and breakdown: adenosine and response to ischemia. In: Opie LH, editor. The heart: physiology and metabolism. New York: Raven Press; 1991. p. 247-74.

20. Ter Keurs HE, Bucx JJ, Harmsen E, Ed Tombe PP, Leijendekker WJ. Ischemic, high-energy phosphate metabolism and sarcomere dynamics in myocardium. In: de Jong JW, editor. Myocardial energy metabolism. Dordrecht, Netherlands: Martinus Nijhoff; 1988. p. $181-94$

21. Tian G, Xiang B, Butler KW, Calafiore AM, Mezzetti A, Salerno TA, Deslauriers R. A ${ }^{31} \mathrm{P}$ nuclear magnetic resonance study of intermittent warm blood cardioplegia. J Thorac Cardiovasc Surg. 1995;109:1155-63.

22. Lojda Z. Studies on dipeptidyl(amino)peptidase IV (glycyl-proline naphthylamidase). Histochemistry. 1979;59:153-66.

23. Tian G, Shen JF, Dai G, Sun J, Xiang B, Luo Z, et al. An interleaved T1-T2* imaging sequence for assessing myocardial injury. J Cardiovasc Magn Reson 1999;1:145-51.

24. Gleason TC, Staelin R. A proposal for handling missing data. Psychometrika. 1975;40:229-52.

25. Mühler A. Assessment of myocardial perfusion using contrast-enhanced MR imaging: current status and future developments. MAGMA. 1995;3:21-33.

26. Kennan RR, Zhong J, Gore JC. Intravascular susceptibility contrast mechanisms in tissue. Magn Reson Med. 1994;31:9-21.

27. Kupriyanov VV, St Jean M, Xiang B, Butler KW, Deslauriers R. Contractile dysfunction caused by normothermic ischemia and $\mathrm{KCl}$ arrest in the isolated pig heart: a ${ }^{31} \mathrm{P}$ NMR study. $\mathrm{J}$ Mol Cell Cardiol. 1995;27:1715-30

28. Kupriyanov VV, Shaw RA, Xiang B, Mantsch H, Deslauriers R. Oxygen regulation of energy metabolism in isolated pig hearts: a near-IR spectroscopy study. J Mol Cell Cardiol. 1997;29:2431-9.

29. Tian G, Xiang B, Dai G, Lindsay WG, Sun J, Shen JF, et al. The effects of retrograde cardioplegia technique on myocardial perfusion and energy metabolism: a magnetic resonance imaging and localized phosphorus 31 spectroscopy study in isolated pig hearts. $J$ Thorac Cardiovasc Surg. 2000;120:544-51.

30. Ardehali A, Laks H, Drinkwater DC, Gates RN, Kaczer E. Ventricular effluent of retrograde cardioplegia in human hearts has traverse capillary beds. Ann Thorac Surg. 1995;60:78-83. 